\title{
Vibrational Analysis of Initially Stressed Carbon Nanotubes
}

\author{
M.M. SELIM* \\ Department of Natural and Applied Sciences, Al-Aflaj Community College, Al-Kharj University \\ Al-Aflaj 710-11912, Saudi Arabia
}

(Received May 26, 2010; in final form February 6, 2011)

\begin{abstract}
In this paper an analytical method to investigate vibrational analysis of double-walled carbon nanotubes under initial compression stress. Simplified Flügge shell equations of motion are proposed as the governing equations of vibration for the carbon nanotubes. The nanotube structures are treated within the multilayer thin shell approximation with the elastic properties taken to be those of the graphene sheet. Vibration frequency equations in double wall carbon nanotubes are described through the effects of initial compression stresses. We investigate the influence of initial compression stress on the vibrational characteristics of carbon nanotubes based on the proposed theoretical approach. In this study, we consider the double-walled nanotubes with an inner diameter of $2.2 \mathrm{~nm}$ and an outer diameter of $3.0 \mathrm{~nm}$. The theoretical investigation may give a useful reference for potential application and design of nanoelectronics and nanodevices.
\end{abstract}

PACS: $45.10 .+-\mathrm{b}$

\section{Introduction}

The number of publications on carbon nanotubes (CNTs) has grown very quickly since CNTs were discovered by Iijima [1] more than a decade ago, as evidenced by several recent review articles on mechanical behavior [2] and [3], fabrication [4] and [5] and applications [6-10] of such materials. Vibrations of CNTs are of considerable importance in a number of nanomechanical devices such as oscillators, charge detectors, clocks, field emission devices and sensors. In addition, CNT vibrations occur during certain manufacturing processes (e.g., ultrasonication in nanocomposite processing) and as part of some nondestructive evaluation processes (e.g., the Raman spectroscopy). Electron microscope observations of vibrating CNTs have also been used to indirectly and nondestructively determine the effective elastic moduli and other aspects of mechanical behavior of the CNTs. Microwave excitation has been found to cause intense heating of CNTs. So there is considerable motivation for studying vibration characteristics of CNTs. The CNTs acting as basic elements of nanostructures often occur in initial stresses due to thermal stress, mismatch between different materials or initially external axial load. Recently, some researches have taken the effect of initial stress on the wave propagation in CNTs [11-15] to understand the dynamic behavior of CNTs. For the effect of initial stress on the wave propagation of CNTs, prior studies presented the elastic wave solution obtained from

* e-mail: msalim@ksu.edu.sa the Bernoulli-Euler beam and the Timoshenko beam models. The effect of initial stress was limited to one-dimensional model since CNTs are modeled as beams. The Euler beam model ignored the influence of rotary and shear deformations on transverse wave propagation in CNTs. Wang and Varadan [16] have presented the elastic wave solution obtained from the Euler-Bernoulli beam and Timoshenko beam models. They reported that the comparison between the two models could be inappropriate on the terahertz frequency range. This suggested that the Timoshenko beam model should be employed in analyzing the wave propagation for the high frequency range.

Recently, the vibration of multi-wall carbon nanotubes (MWCNTs) and wave propagation of double-wall carbon nanotubes (DWCNTs) have been studied based on the Flügge shell equation $[17,18]$. These works show that the CNTs have the vibration and sound wave frequency over terahertz because of their nanoscale, which opens a new topic on wave characteristics.

The purpose of this article is to study effects of initial compression stress on vibrational analysis of double-walled carbon nanotubes. The analysis is based on the Flügge shell equation. As the wave numbers are the roots of an eighth order polynomial, Derive program version 6 was used to derive algebraic expressions for these roots and to simplify the algebraic expressions via a series of substitutions. The expressions presented here are valid for circumferential modes $n=0$ and $n=1$. The results are shown graphically for different values of initial compression stress parameter. 


\section{Governing equations}

The cylindrical coordinates system used for describing the wave propagation in the tubes under initial compression stress is defined in Fig. 1. Approximate Flügge shell equations are proposed as the governing equations of the problem [19]. In the derivation of Flügge's equations, the following assumptions for cylindrical shells are made:

(1) All points that lie on a normal to a middle surface before deformation do the same after deformation.

(2) Displacements are small compared to the shell thickness.

(3) The normal stresses in the thickness direction are negligible (planar state of stress).

In particular, the first assumption may not correspond to reality in the neighborhood of the shell boundaries. However, this fact will not be considered here.

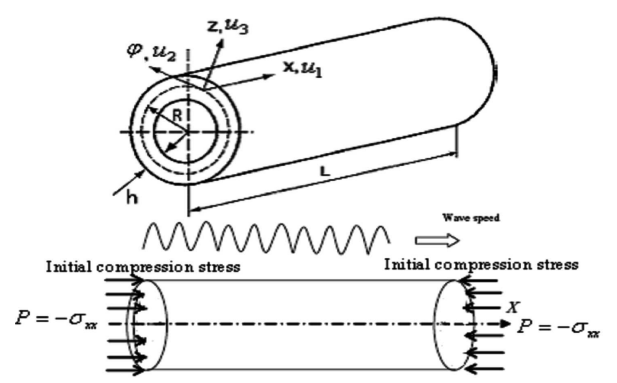

Fig. 1. Geometry of the tube with coordinate system.

In the sequel, the $x$ coordinate is taken in the axial direction of the shell, where the $\varphi$ and $z$ coordinates are in the circumferential and radial directions, respectively (Fig. 1). The displacements of the nanotube are defined by $u_{1}, u_{2}$, and $u_{3}$ in the direction of $x, \varphi$ and $z$ axes, respectively. The coordinates $u_{1}$ and $u_{2}$ represent in-plane axial and circumferential displacements of the tube wall midsurface, respectively, and $u_{3}$ represents the out-of-plane transverse displacement of the tube wall. The CNT has a thickness of $h$, radius of $R$ and length of $L$.

Based on the assumption stated above and using the coordinate system defined in Fig. 1, the Flügge type basic equations of motion are proposed as the governing equations of the wave propagation under initial compression stress $\left(\sigma_{x x}=-P\right)$ in CNTs. The equations of motion for the nanotube can be written by [20]:

$$
\begin{aligned}
& {\left[\begin{array}{ccc}
L_{11}+\alpha_{11} & L_{12}+\alpha_{12} & L_{13}+\alpha_{13} \\
L_{12}+\alpha_{21} & L_{22}+\alpha_{22} & L_{23}+\alpha_{23} \\
-L_{13}+\alpha_{31} & -L_{23}+\alpha_{32} & L_{33}+\alpha_{33}
\end{array}\right]\left[\begin{array}{l}
u_{1} \\
u_{2} \\
u_{3}
\end{array}\right]} \\
& =\left[\begin{array}{c}
0 \\
0 \\
-\beta \hat{P}
\end{array}\right],
\end{aligned}
$$

where $L_{i j}(i, j=1,2,3)$ are the differential operators with respect to $x$ and $\theta$, given by

$$
\begin{aligned}
L_{11} & =\frac{\partial^{2}}{\partial x^{2}}+\frac{1-\nu}{2 R^{2}} \frac{\partial^{2}}{\partial \varphi^{2}}-\beta \rho h \frac{\partial^{2}}{\partial t^{2}} \\
L_{12} & =\frac{1+\nu}{2 R} \frac{\partial^{2}}{\partial x \partial \varphi}, \quad L_{13}=\frac{\nu}{R} \frac{\partial}{\partial x} \\
L_{22} & =\frac{1-\nu}{2} \frac{\partial^{2}}{\partial x^{2}}+\frac{1}{R^{2}} \frac{\partial^{2}}{\partial \varphi^{2}} \\
+ & \left((1-\nu) \frac{\partial^{2}}{\partial x^{2}}+\frac{1}{R^{2}} \frac{\partial^{2}}{\partial \varphi^{2}}\right)-\beta \rho h \frac{\partial^{2}}{\partial t^{2}}, \\
L_{23} & =\frac{1}{R^{2}} \frac{\partial}{\partial \varphi}-\sigma\left((2-\nu) \frac{\partial^{3}}{\partial x^{2} \partial \varphi}+\frac{1}{R^{2}} \frac{\partial^{3}}{\partial \varphi^{3}}\right) \\
L_{33} & =-\frac{1}{R^{2}}-\sigma\left(R^{2} \frac{\partial^{4}}{\partial x^{4}}+2 \frac{\partial^{4}}{\partial x^{2} \partial \varphi^{2}}+\frac{1}{R^{2}} \frac{\partial^{4}}{\partial \varphi^{4}}\right) \\
- & \beta \rho h \frac{\partial^{2}}{\partial t^{2}},
\end{aligned}
$$

and

$$
\begin{aligned}
\alpha_{11}=\zeta_{x}(1-\nu) \frac{\partial^{2}}{\partial x^{2}}, \alpha_{12}=\frac{\zeta_{x}(2 h-1)(1-\nu)}{2 R} \frac{\partial^{2}}{\partial x \partial \varphi}, \\
\alpha_{13}=\frac{\zeta_{x}(1-\nu)}{R}\left(\frac{\partial}{\partial x}+\frac{1}{2 R h} \frac{\partial^{2}}{\partial \varphi^{2}}\right), \\
\alpha_{21}=\zeta_{x}(1-\nu) \frac{\partial^{2}}{\partial x \partial \varphi}, \alpha_{22}=\frac{\zeta_{x}(1-\nu)}{2 h} \frac{\partial^{2}}{\partial x^{2}}, \\
\alpha_{23}=\frac{\zeta_{x}(1-\nu)}{2 h R}\left(\frac{\partial^{2}}{\partial x \partial \varphi}-\frac{h^{3}}{6 R} \frac{\partial^{3}}{\partial x^{2} \partial \varphi}\right), \\
\alpha_{31}=\frac{\zeta_{x}(1-\nu)}{R} \frac{\partial}{\partial x}, \alpha_{32}=\frac{\zeta_{x} h^{2}(1-\nu)}{12 R} \frac{\partial^{3}}{\partial x^{2} \partial \varphi}, \\
\alpha_{33}=\frac{\zeta_{x}(1-\nu)}{2 h}\left(\frac{\partial^{2}}{\partial x^{2}}+\frac{h^{3}}{6} \frac{\partial^{4}}{\partial x^{4}}+\frac{h^{3}}{3 R^{2}} \frac{\partial^{3}}{\partial x^{2} \partial \varphi}\right),(3)
\end{aligned}
$$

$\sigma=\frac{h^{2}}{12 R^{2}}$ is the non-dimensional thickness parameter, and $\beta=\frac{\left(1-\nu^{2}\right)}{E h}, \zeta_{x}=\frac{P(1+\nu)}{E}, \rho$ is the mass density, $E$ is the elastic modulus, $\nu$ is the Poisson ratio, $P$ are the initial compression stresses, and $\hat{P}$ is the pressure between two adjacent nanotubes (mainly due to the van der Waals (vdW) interaction).

Equation (1) represents a set of three expressions as

$$
\begin{aligned}
& D_{11} u_{1}+D_{12} u_{2}+D_{13} u_{3}=0, \\
& D_{21} u_{1}+D_{22} u_{2}+D_{23} u_{3}=0, \\
& D_{31} u_{1}+D_{32} u_{2}+D_{33} u_{3}+\beta \hat{P}=0,
\end{aligned}
$$

where

$$
\begin{aligned}
& D_{11}=L_{11}+\alpha_{11}, D_{12}=L_{12}+\alpha_{12}, D_{13}=L_{13}+\alpha_{13}, \\
& D_{21}=L_{12}+\alpha_{21}, D_{22}=L_{22}+\alpha_{22}, D_{23}=L_{23}+\alpha_{23}, \\
& D_{31}=-L_{13}+\alpha_{31}, \quad D_{32}=-L_{23}+\alpha_{32} \\
& D_{33}=L_{33}+\alpha_{33} .
\end{aligned}
$$

Eliminating $u_{1}$ and $u_{2}$ from the above equation, we get 
where

$$
D_{1} u_{3}+D_{2} \hat{P}=0 \text {. }
$$

$$
\begin{aligned}
& D_{1}=D_{32}\left(D_{11} D_{23}-D_{21} D_{13}\right)+D_{31}\left(D_{13} D_{22}\right. \\
& \left.\quad-D_{12} D_{23}\right)+D_{33}\left(D_{12} D_{21}-D_{11} D_{22}\right), \\
& D_{2}=\left(D_{21} D_{12}-D_{11} D_{22}\right) .
\end{aligned}
$$

\section{Particular case}

When the effect of initial compression stresses is absent $\left(\zeta_{x}=0\right)$ (i.e. $\left.\alpha_{i j}=0, i, j=1,2,3\right)$ Eq. (2) becomes

$$
\left[\begin{array}{ccc}
L_{11} & L_{12} & L_{13} \\
L_{12} & L_{22} & L_{23} \\
-L_{13} & -L_{23} & L_{33}
\end{array}\right]\left[\begin{array}{l}
u_{1} \\
u_{2} \\
u_{3}
\end{array}\right]=\left[\begin{array}{c}
0 \\
0 \\
-\hat{P} \beta
\end{array}\right]
$$

which agrees with the result of Natsuki et al. [21].

\section{Vibrational frequency}

To examine the effect of compression initial stresses on vibrational frequency of DWNTs, let us assume the displacement of simply supported DWNTs for the harmonic vibration as

$$
u_{3 j}=A_{j} \cos (n \varphi) \sin \left(\frac{m \pi x}{L}\right) \exp (\mathrm{i} \omega t), j=1,2,
$$

where $A_{j}(j=1,2)$ represents the amplitude of vibration in the inner and outer nanotubes. $\omega$ is the circular frequency of wave. The modal $m$ and $n$ denote the number of axial and the circumferential waves, respectively, characterizing the vibrational mode of CNTs. The amplitude ratio of the inner to the outer tubes is defined as $A_{1} / A_{2}$.
From the geometric structures, a DWCNT can be regarded as nested SWCNTs. The pressure $(\hat{P})$ between two adjacent nanotubes is mainly due to the van der Waals interaction. Thus for small-deflection linear vibration, the interaction pressure at any point between two adjacent tubes was modeled by a linear function of the deflection jump at that point [22]. In terms of the above model of the vdW interaction, the interaction pressure $\hat{P}$ in Eq. (6) can be given by

$$
\hat{P}=\Delta u \text {, }
$$

where $\Delta u=c\left(u_{32}-u_{31}\right)$ is the deflection jump due to deformation

$$
\begin{aligned}
& \hat{P}_{1}=c\left(u_{32}-u_{31}\right), \\
& \hat{P}_{2}=-c\left(u_{32}-u_{31}\right),
\end{aligned}
$$

where $c$ is the vdW interaction coefficient, approximately expressed as [23]:

$$
c=\frac{200\left[\mathrm{erg} / \mathrm{cm}^{2}\right]}{0.16 d^{2}}, \quad d=1.42[\mathrm{~nm}] .
$$

From Eq. (11) in Eq. (6), the coupled equations of the wave speed in double-walled nanotubes can be written as

$$
\begin{aligned}
& D_{1}^{\mathrm{i}} u_{31}+D_{2}^{\mathrm{i}}\left[\Omega\left(u_{32}-u_{31}\right)\right]=0, \\
& D_{1}^{\mathrm{o}} u_{32}+D_{2}^{\mathrm{o}}\left[-\Omega\left(u_{32}-u_{31}\right)\right]=0,
\end{aligned}
$$

where $D_{1}^{\mathrm{i}}$ and $D_{2}^{\mathrm{i}}$ are the differential operators for the inner tube, $D_{1}^{\mathrm{o}}$ and $D_{2}^{\mathrm{o}}$ are the differential operators for the outer tube defined in Eq. (7) and $\Omega=c \beta$. Substituting Eq. (9) into Eq. (13), the vibrational frequency in DWNTs can be determined by solving the two equations, which have only nontrivial solution at

$$
\left|\begin{array}{cc}
D_{1}^{\mathrm{i}}\left(\omega, m, n, \zeta_{x}\right)-\Omega D_{2}^{\mathrm{i}}\left(\omega, m, n, \zeta_{x}\right) & \Omega D_{2}^{\mathrm{i}}\left(\omega, m, n, \zeta_{x}\right) \\
\Omega D_{2}^{\mathrm{o}}\left(\omega, m, n, \zeta_{x}\right) & D_{1}^{\mathrm{o}}\left(\omega, m, n, \zeta_{x}\right)-\Omega D_{2}^{\mathrm{o}}\left(\omega, m, n, \zeta_{x}\right)
\end{array}\right|=0 .
$$

The amplitude ratio of the inner nanotube to the outer nanotube is given by

$$
\frac{A_{1}}{A_{2}}=\frac{\Omega D_{2}^{\mathrm{i}}\left(\omega, m, n, \zeta_{x}\right)}{\Omega D_{2}^{\mathrm{i}}\left(\omega, m, n, \zeta_{x}\right)-D_{1}^{\mathrm{i}}\left(\omega, m, n, \zeta_{x}\right)} .
$$

\section{Numerical results and discussion}

The purpose of numerical computation is to examine the effect of compression of initial stresses on vibrational frequency of DWNTs. In order to perform numerical calculation, the CNT to be considered has the thickness of graphene sheet with $0.34 \mathrm{~nm}$, elastic modulus of $E=1.095 \mathrm{TPa}$, and Poisson's ratio of $\nu=0.19$. The mass density of CNTs is of $\rho=1.3 \mathrm{~g} / \mathrm{cm}^{3}$. The DWCNT consisted of the $(40,0)$ and $(50,0)$ zigzag tubes. The inner and outer diameters of DWNTs are $2.0 \mathrm{~nm}$ and $3.0 \mathrm{~nm}$, respectively.

What follows is to analyze linear vibration behavior of a DWCNT subjected to axially initial compressed stresses $\left(\sigma_{x x}=-P\right)$. For the following solution we will discuss the two modes, symmetrical $(n=0)$ and asymmetrical $(n=1)$ to show the effects of initial stresses on vibrational frequency of DWNTs. The influences of wave numbers on the linear vibration pattern at different values of stress parameters $\left(\zeta_{x}=0.0,0.01,0.025\right)$ are examined and the results are shown as follows.

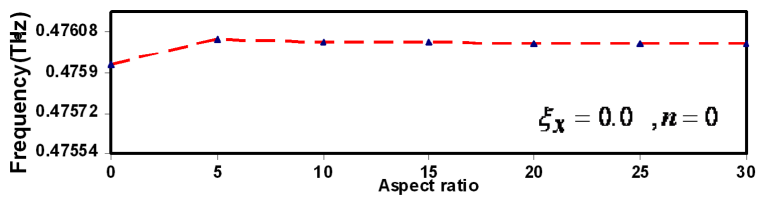

Fig. 2. Vibrational frequency of DWNTs as a function of the aspect ratio without initial stress $\left(\zeta_{x}=0.0\right)$ for $n=0$.

Figure 2 shows the natural frequency of DWNTs as a function of the aspect ratio without initial stress $\left(\zeta_{x}=\right.$ 0.0 ) for $n=0$ In order to examine the influence of the initial compression stresses on the vibrational frequency 


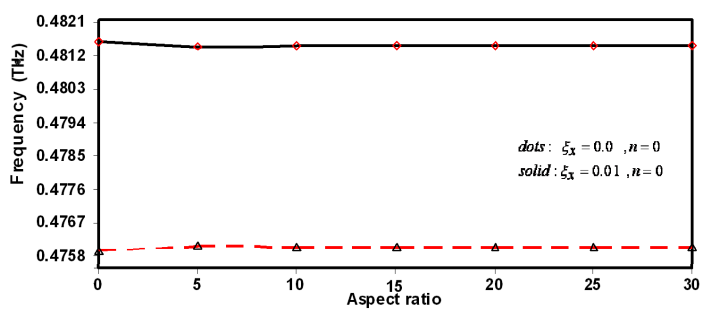

Fig. 3. Influence of initial compression stress $\left(\zeta_{x}=\right.$ 0.01 ) on vibrational frequency of DWNTs with different aspect ratio for $n=0$.

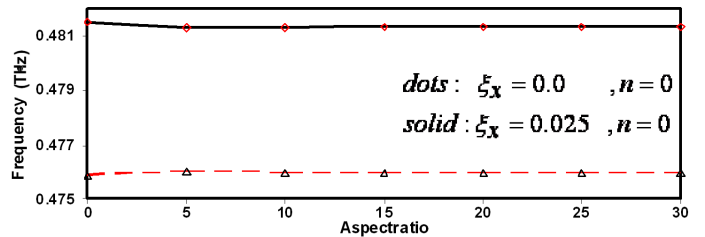

Fig. 4. Influence of initial compression stress $\left(\zeta_{x}=\right.$ $0.025)$ on vibrational frequency of DWNTs with different aspect ratio for $n=0$.

of DWCNTs, the results under initial compression stress $\zeta_{x}=0.01, \zeta_{x}=0.025$ are compared with corresponding ones without initial stress $\left(\zeta_{x}=0.0\right)$ in Figs. 3 and 4 , respectively. Comparisons of Figs. 3 and 4 with Fig. 2 show that the change tendency of the vibrational frequency in double-walled CNTs with initial compression stresses $\zeta_{x}=0.01$ is similar to those with initial compression stresses $\zeta_{x}=0.025$, but a change is observed in these figures compared with Fig. 2 for initial stress-free $\left(\zeta_{x}=0.0\right.$ at $\left.n=0\right)$. It is noted that from Figs. 3 and 4 it follows that as the value of initial stress parameter $\left(\zeta_{x}\right)$ increases, frequencies increase.

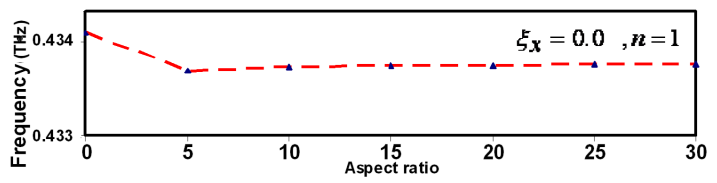

Fig. 5. Vibrational frequency of DWNTs as a function of the aspect ratio without initial stress $\left(\zeta_{x}=0.0\right)$ for $n=1$.

Figures 5-7 show the effect of initial compression stresses with different aspect ratio for DWCNTs for $n=$ 1. In Fig. 5, vibrational frequency of DWNTs as a function of the aspect ratio without initial stress $\left(\zeta_{x}=0.0\right)$ for $n=1$ is plotted. Comparisons of Figs. 6 and 7 with Fig. 5 show that the natural frequency in double-walled CNTs with initial compression stresses $\zeta_{x}=0.01$ and $\zeta_{x}=0.025$ are sensitive to the present initial compression stresses. It can be seen from Fig. 7 that the frequencies decrease as initial stress parameter $\left(\zeta_{x}\right)$ increases. This results are different from the results obtained at $n=0$.

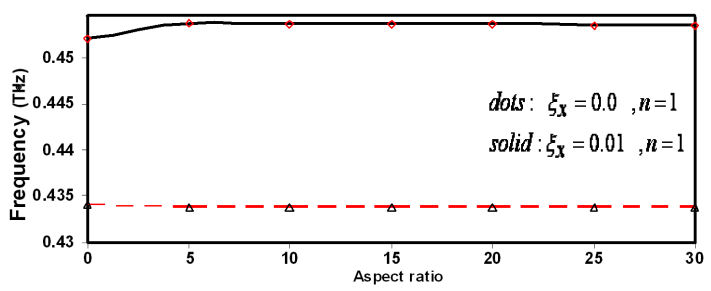

Fig. 6. Influence of initial compression stress $\left(\zeta_{x}=\right.$ $0.01)$ on vibrational frequency of DWNTs with different aspect ratio for $n=1$.

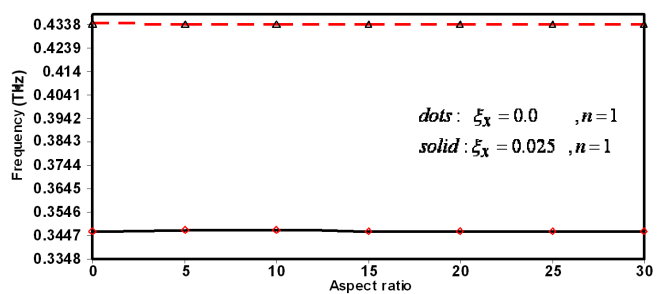

Fig. 7. Influence of initial compression stress $\left(\zeta_{x}=\right.$ 0.025 ) on vibrational frequency of DWNTs with different aspect ratio for $n=1$.

\section{Conclusion}

This paper studies the effects of initial compression stress on vibrational frequency of DWNTs based on the Flügge shell equation. It is shown that the vibrational frequency is sensitive to the compression stress especially for the lower frequencies $(m=0.5)$. The investigation presented may be helpful in the application of CNTs, such as high-frequency oscillators and mechanical sensors.

\section{Acknowledgments}

The author wish to acknowledge support provided by the deanship of scientific research of Al-Kharj University and the Aflaj community college. Also, the author are thankful to Dr. Rashed Al-Rushood (dean of the Aflaj community college) for his supporting and encouragement during this work.

\section{References}

[1] S. Iijima, Nature 354, 56 (1991).

[2] D. Qian, J.G. Wagner, W.K. Liu, M.F. Yu, R.S. Ruoff, Appl. Mech. Rev. 55, 495 (2002).

[3] E.T. Thostenson, Z. Ren, T.W. Chou, Compos. Sci. Technol. 61, 1899 (2001).

[4] H.J. Dai, Surf. Sci. 500, 218 (2002).

[5] J. Liu, S.S. Fan, H.J. Dai, MRS Bull. 29, 244 (2004).

[6] P.G. Collins, P. Avouris, Sci. Am. 283, 62 (2000).

[7] P. Avouris, J. Appenzeller, R. Martel, S.J. Wind, Proc. IEEE 91, 1772 (2003).

[8] K. Tsukagoshi, N. Yoneya, S. Uryu, Y. Aoyagi, A. Kanda, Y. Ootuka, B.W. Alphenaar, Physica B 323, 107 (2002). 
[9] R.H. Baughman, A.A. Zakhidov, W.A. de Heer, Science 297, 787 (2002).

[10] W.B. Choi, E. Bae, D. Kang, S. Chae, B. Cheong, J. Ko, , E. Lee, W.B. Park, Nanotechnology 15, S512 (2004).

[11] Y. Zhang, G. Liu, X. Han, Phys. Lett. A 340, 258 (2005).

[12] H. Zhang, X. Wang, Nanotechnology 17, 45 (2006).

[13] X. Wang, H. Cai, Acta Mater. 54, 2067 (2006).

[14] K. Liu, C. Sun, Solid State Commun. 143, 202 (2007).

[15] X. Chen, X. Wang, Phys. Scr. 78, 015601 (2008).

[16] Q. Wang, V.K. Varadan, Int. J. Solids Struct. 43, 254 (2006).
[17] C.Y. Wang, C.Q. Ru, A. Mioduchowski, Phys. Rev. $B$ 72, 075414 (2005).

[18] T. Natsuki, M. Endo, H. Tsuda, J. Appl. Phys. 99, 034311 (2006).

[19] W. Flügge, Statik und Dynamik der Schalen, Springer, Berlin 1934.

[20] M.M. Selim, S. Abi, K. Harigaya, Eur. Phys. J. B 69, 523 (2009).

[21] T. Natsuki, Q.-Q. Ni, M. Endo, J. Appl. Phys. A 90, 441 (2008).

[22] J. Yoon, C.Q. Ru, A. Mioduchowski, Compos. Sci. Technol. 63, 1533 (2003).

[23] C.Q. Ru, Phys. Rev. B 62, 16962 (2000). 